\title{
Multiphysics Numerical Modeling of a Fin and Tube Heat Exchanger
}

\author{
Shobhana Singh Kim Sørensen Thomas Condra \\ Department of Energy Technology, Aalborg University, Denmark \\ ssidet.aau.dk, ksodet.aau.dk, tcdet.aau.dk
}

\begin{abstract}
In the present research work, a modeling effort to predict the performance of a liquid-gas type fin and tube heat exchanger design is made. Three dimensional (3D) steady state numerical model is developed using commercial software COMSOL Multiphysics based on finite element method (FEM). For the purposes here, only gas flowing over the fin side is simulated assuming constant inner tube wall temperature. The study couples conjugate heat transfer mechanism with turbulent flow in order to describe the temperature and velocity profile. In addition, performance characteristics of the heat exchanger design in terms of heat transfer and pressure loss are determined by parameters such as overall heat transfer coefficient, Colburn $j$-factor, flow resistance factor, and efficiency index. The model provides useful insights necessary for optimization of heat exchanger design.
\end{abstract}

Keywords: Fin and tube heat exchanger, turbulent flow, conjugate heat transfer, numerical modeling, COMSOL

\section{Introduction}

Heat exchangers are commonly used thermal energy systems for a variety of applications. They are the key components in waste heat recovery technologies. Selection of suitable heat exchanger design is essential to achieve maximum efficiency of the system. However, the thermal performance of liquid-gas heat exchangers is greatly influenced by the high thermal resistance on gas-side which account for more than $80 \%$ of the total thermal resistance [Malapure et al., 2007; Kundu and Lee, 2011; Yaïci et al., 2014]. Over the past decade, fin and tube heat exchangers have been given a considerable attention among other available heat exchanger designs due to extended heat transfer surface area and ease of manufacturing [Rocha et al., 1997; Jang and Yang, 1998; Matos et al., 2004; Mon and Gross, 2004; Erek et al., 2005; Ibrahim and Gomma, 2009; Taler and Ocłon, 2014; Hatami et al. 2014a; Lopata and Ocłon, 2015].

Several numerical investigations of fin and tube heat exchangers for different applications including waste recovery systems are available in the literature. Chen et al. (2007) performed a numerical simulation of laminar flow with the finite-volume method in a finned oval tube heat exchanger and studied conjugate heat transfer along with flow patterns and pressure distributions. Hatami et al. (2014b) numerically studied two cases for fin type heat exchangers in engines for exhaust waste heat recovery and concluded that recovered heat can be improved by increasing the fin numbers and length where maximum heat recovery occurs. Vortex generators of different types on the fin surface of the heat exchangers have also been studied extensively as an effective method to improve the air side heat transfer performance [Fiebig, 1995; Jacobi and Shah, 1995; Chang et al., 2009; Habchi et al. 2012; Gong et al., 2015; Li et al., 2015]. Chu et al. (2009) employed 3D numerical simulations to investigate the heat transfer characteristics and flow structure in fullscale fin-and-oval-tube heat exchangers with longitudinal vortex generators. Moreover, three dimensional simulations were accomplished by Borrajo-Peláez et al. (2010) to compare both air side and water side model and evaluate the performance through non-dimensional parameters: the air side Nusselt number and friction factor.

In most of the applications, various fin patterns (such as wavy, louver, slit, and convex-louver) are adopted in order to improve the performance of heat exchanger [Wang et al., 1999; Tao et al., 2007; Tang et al., 2009; Kundu and Lee, 2011]. Malapure et al. (2007) investigated fluid flow and heat transfer characteristics of louvered fins and flat tube with single and double row of tubes with in compact heat exchangers by 3D numerical simulations. Tao et al. (2007) also performed 3D numerical simulations for laminar flow of wavy fin-and-tube heat exchangers and reported that the fin efficiency at the inlet region of wavy fins is larger than that of plain plate fins. Most recently, Cobian- Iñiguez et al. (2015) introduced a methodology of analysis based on local and global energy balances to determine the hydrodynamic and heat transfer characteristics. The authors also emphasized on analyzing the parameters such as fin spacing, tube diameter and alignment, and flow velocity for designing efficient heat exchangers.

Innovative fin and tube heat exchanger designs always offer scope to explore possible opportunities for overall performance improvement. With this view in mind, present paper mainly focusses on predicting the performance of a fin and tube heat exchanger design by 
utilizing multiphysics approach. 3D steady state numerical model using FEM is developed to predict the heat transfer characteristics as well as flow profile for a given fin and tube heat exchanger design. Heat exchanger performance characteristics, such as overall heat transfer coefficient, Colburn j-factor, flow resistance factor and efficiency index are estimated.

\section{Fin and tube heat exchanger design}

The heat exchanger design analyzed in the present work is of liquid-gas fin and tube type with plain fins and circular tubes. The typical configuration of the fin and tube exchanger is shown in Figure 1. The tubes are arranged in an in-line manner in a flow direction at fixed distance to each other, and fins are attached on a set of two tubes from both sides leaving a gap in between. It should be noted that the contact surface between the fins and tubes is assumed to be perfectly conducting (i.e. no gaps due to roughness). Figure 2 represents the schematic diagram of the single unit of heat exchanger design with specified dimensions as described in Table 1. The single unit design includes two circular tubes and two fins which on repetitive arrangement at fixed distance builds the complete fin and tube heat exchanger configuration for desired applications. The conjugate heat transfer phenomenon includes simultaneous convective heat transfer from the flowing fluid to the fin surface and tube outer wall surface, and conductive heat transfer from the outer wall to inner wall of the tube as well as through fin to tube.

\section{Model development}

\subsection{Computational geometry}

Numerical model in the present study entails a simple 3D computational geometry of the fin and tube heat exchanger design. Having symmetric geometry of the heat exchanger, we simulated only one half of the fin and tube heat exchanger unit. This keeps the simplicity of the model and reduces computational time as well as cost. In applications where evaporation or condensation of the fluid takes place inside the tubes, average temperature of inner tube wall temperature does not change or remains constant. For that purpose, only the fluid flowing over the fins outside the tubes is modeled in the present work. The 3D computational geometry consists of three distinct domains: gas domain, fin domain, and tube domain as shown in Figure 3. The dimensions of the heat exchanger geometry is chosen according to the given heat exchanger prototype design (Figure 1). To capture flow profile at the inlet, outlet and both sides of the heat exchanger geometry, gas domain is simulated with length 1.03 times of fin length $\left(L_{f}\right)$ and width 1.18 times of fin width $\left(W_{f}\right)$.

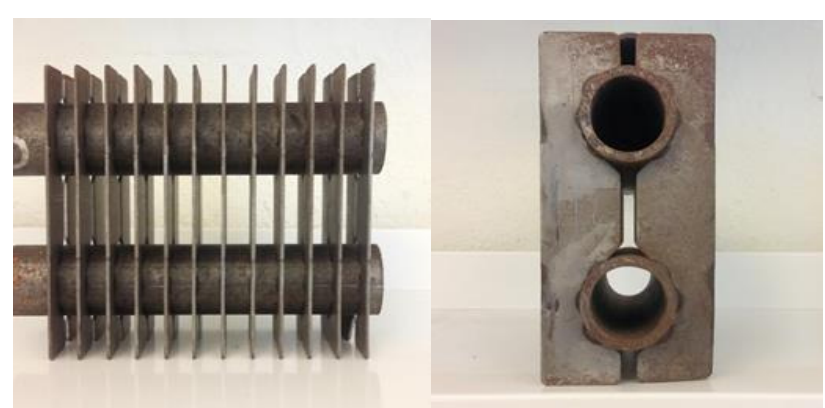

Figure 1. Pictorial view of the fin and tube heat exchanger prototype.
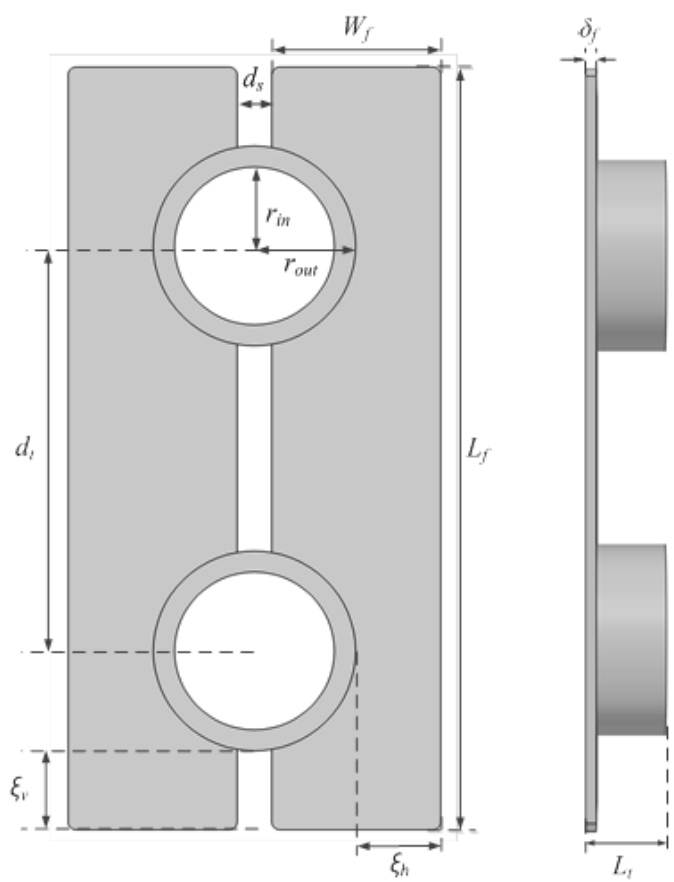

Figure 2. Schematic of the single unit of fin and tube heat exchanger.

Table 1. Design parameters used in numerical model

\begin{tabular}{|l|c|c|c|}
\hline Description & Symbol & Value & Unit \\
\hline Length of the gas domain & $L_{g}$ & 150.0 & $\mathrm{~mm}$ \\
\hline Width of the gas domain & $W_{g}$ & 37.5 & $\mathrm{~mm}$ \\
\hline Length of the fin & $L_{f}$ & 145.0 & $\mathrm{~mm}$ \\
\hline Width of the fin & $W_{f}$ & 31.75 & $\mathrm{~mm}$ \\
\hline Thickness of the fin & $\delta_{f}$ & 2.0 & $\mathrm{~mm}$ \\
\hline $\begin{array}{l}\text { Distance between two fins } \\
\text { (center to center) }\end{array}$ & $d_{f}$ & 13.0 & $\mathrm{~mm}$ \\
\hline Thickness of the gap in fin & $d_{s}$ & 6.5 & $\mathrm{~mm}$ \\
\hline Inner radius of the tube & $r_{i n}$ & 15.0 & $\mathrm{~mm}$ \\
\hline Outer radius of the tube & $r_{\text {out }}$ & 19.0 & $\mathrm{~mm}$ \\
\hline Length of the tube & $L_{t}$ & 15.0 & $\mathrm{~mm}$ \\
\hline $\begin{array}{l}\text { Tube spacing (center to } \\
\text { center) }\end{array}$ & $d_{t}$ & 77.0 & $\mathrm{~mm}$ \\
\hline Tube pitch (vertical) & $\xi_{v}$ & 15.0 & $\mathrm{~mm}$ \\
\hline Tube pitch (horizontal) & $\xi_{h}$ & 16.0 & $\mathrm{~mm}$ \\
\hline
\end{tabular}




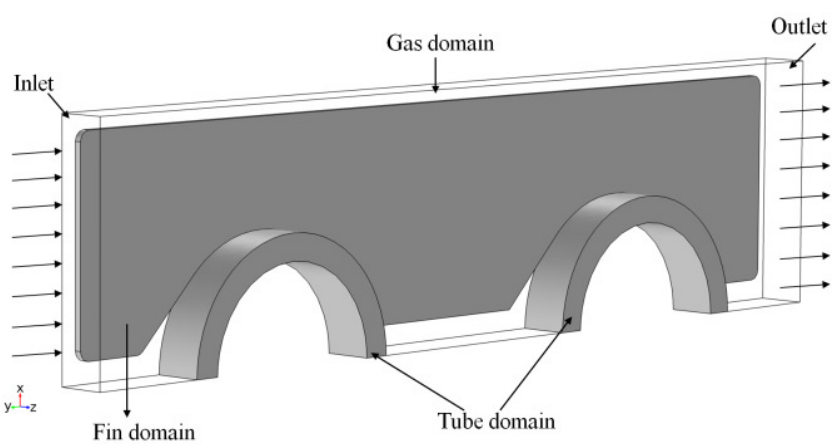

Figure 3. Domains and boundaries of computational geometry used in the numerical model.

Furthermore, gas domain represents the inlet and outlet boundary planes through which hot fluid enters and leaves the domain, respectively. The flow of gas in the gas domain is in negative y-direction. Fin and tube are solid domains that are assumed to be made of steel and the gas domain is simulated as ideal gas with constant air properties.

\subsection{Governing Equations}

The present 3D numerical model of a fin and tube heat exchanger design includes conjugate heat transfer that corresponds to the combination of heat transfer phenomenon in fin, tube and gas domains simultaneously. To simplify the model and computation, following assumptions are made:

- Steady state flow and heat transfer.

- Incompressible gas flow.

- Constant inner tube wall temperature.

- Thermal contact resistance is negligible.

- Constant material properties.

- No periodic boundary condition.

Considering the turbulent nature of the flow in the gas domain, $\mathrm{k}-\varepsilon$ turbulence model is used to solve the Reynolds-Averaged Navier-Stokes (RANS) equations for the averaged velocity and pressure fields in the gas flow domain. $\mathrm{k}-\varepsilon$ model is chosen as it performs well for most of the flow problems around complex geometries and can deliver an accurate enough result with good convergence rate and relatively low memory requirements.

The coupling of mass balance and the momentum balance for the flow in the gas domain is governed by RANS equations [Kuzmin et al., 2007; Hatami et al., 2014c] which are expressed as:

$\nabla \cdot \mathbf{u}=0$

$\rho(\mathbf{u} \cdot \nabla) \mathbf{u}=\nabla \cdot\left[-p \mathbf{I}+\mu\left(\nabla \mathbf{u}+(\nabla \mathbf{u})^{T}\right)\right]+\mathbf{F}$

where, u $[\mathrm{m} / \mathrm{s}]$ is the averaged velocity vector, $\rho$ $\left[\mathrm{kg} / \mathrm{m}^{3}\right]$ and $\mu$ [Pa.s] are the density and dynamic viscosity of the gas, respectively.
$p[\mathrm{~Pa}]$ is the averaged pressure, and $\mathbf{F}\left[\mathrm{N} / \mathrm{m}^{3}\right]$ is the body force vector.

The $\mathrm{k}-\varepsilon$ turbulence model also solves the turbulent kinetic energy, $\mathrm{k}\left[\mathrm{m}^{2} / \mathrm{s}^{2}\right]$ and turbulent dissipation rate, $\varepsilon\left[\mathrm{m}^{2} / \mathrm{s}^{3}\right]$. The differential transport equations describing the $\mathrm{k}$ and $\varepsilon$ in the gas domain are:

$$
\begin{aligned}
& \rho \mathbf{u} \cdot \nabla k=\nabla \cdot\left[\left(\mu+\frac{\mu_{T}}{\sigma_{k}}\right) \nabla k\right]+P_{k}-\rho \varepsilon \\
& \rho \mathbf{u} \cdot \nabla \varepsilon=\nabla \cdot\left[\left(\mu+\frac{\mu_{T}}{\sigma_{\varepsilon}}\right) \nabla \varepsilon\right]+C_{\varepsilon 1} \frac{\varepsilon}{k} P_{k}-C_{\varepsilon 2} \frac{\varepsilon^{2}}{k}
\end{aligned}
$$

where, $P_{k}$ is the production term, which is defined as:

$$
P_{k}=\mu_{T}\left[\nabla \mathbf{u}:\left(\nabla \mathbf{u}+(\nabla \mathbf{u})^{T}\right)\right]
$$

The turbulent viscosity, $\mu_{T}$ is modeled as:

$\mu_{T}=\rho C_{\mu} \frac{k^{2}}{\varepsilon}$

where, $C_{\mu}, C_{\varepsilon 1}, C_{\varepsilon 2}, \sigma_{k}$, and $\sigma_{\varepsilon}$ are model constants with values $0.09,1.44,1.92,1.0$ and 1.3 , respectively which are used to numerically solve the model [Wilcox, 1998].

The energy transport in terms of heat transfer within gas domain is driven by both convection and conduction; however, based on the thermal properties of the fluid and the inlet velocity assumed in this study, it is mainly dominated by convection. The differential equation that describes the heat transfer in gas domain and solves for the temperature field is:

$\rho C_{p} \mathbf{u} \cdot \nabla T+\nabla \cdot \mathbf{q}=Q$

where, $\mathbf{q}\left[\mathrm{W} / \mathrm{m}^{2}\right]$ is the heat flux vector and is expressed as: $\mathbf{q}=-k \nabla T . T[\mathrm{~K}]$ is the temperature, $C_{p}$ $[\mathrm{J} /(\mathrm{kg} . \mathrm{K})]$ is the heat capacity at constant pressure, $k$ $[\mathrm{W} /(\mathrm{m} . \mathrm{K})]$ is the thermal conductivity, and $Q\left[\mathrm{~W} / \mathrm{m}^{2}\right]$ is the heat source or sink.

The heat transfer in the fin and tube domain is dominated by conduction and hence governs by Fourier's Law of heat conduction. It is to note that due to small temperature difference and lower emissivity, heat transfer through radiation is neglected. The heat transfer equation in the fin and tube domains also account for additional heat source or sink term and is expresses in the following form of energy conservation:

$$
\rho C_{p} \mathbf{u} \cdot \nabla T+\nabla \cdot \mathbf{q}=Q
$$

The first term represents contribution of translational motion to the heat transfer in solids, which is negligible in this case, hence neglected. Second term is the conductive heat flux vector, which is expressed as: $\mathbf{q}=-k_{s} \nabla T$ where, $k_{s}[\mathrm{~W} /(\mathrm{m} . \mathrm{K})] \quad$ is the thermal conductivity of fin and tube material. 


\subsection{Boundary conditions}

To pose a well-defined problem, we impose suitable boundary conditions to numerically solve the governing equations. Initial condition is simulated by assuming no gas flow in gas domain i.e. the velocity field is set to $0 \mathrm{~m} / \mathrm{s}$ with pressure set to $0 \mathrm{~Pa}$. Furthermore, the temperature of all three domains (fin, tube and gas domains) is set to room temperature at $\mathrm{T}=$ $298.15 \mathrm{~K}$ corresponding to no heat transfer at the initial stage of the simulation.

\subsubsection{Inlet boundary condition}

We set appropriate boundary conditions to define the conjugate heat transfer process in the considered geometry. At the inlet of the flow domain, the temperature boundary condition is set as:

$T=T_{i n}$

The inlet velocity field boundary condition for the flow of fluid in the gas domain is:

$$
\left.\begin{array}{l}
u=0 \\
v=-u_{i n} \\
w=0
\end{array}\right\}
$$

\subsubsection{Wall boundary condition}

The contact surface between fin, tube and gas domains are the boundaries from where the heat flux is exchanged. In the present model, wall function boundary condition is applied which simulates the region near the contact surface where the flow variables have high gradients.

$$
\left.\begin{array}{l}
\mathbf{u} \cdot \mathbf{n}=0 \\
\nabla k \cdot \mathbf{n}=0 \\
\nabla \varepsilon \cdot \mathbf{n}=0
\end{array}\right\}
$$

For the purposes here, the inner tube wall temperature is assumed to be constant and the temperature boundary condition is set as:

$T=T_{w}$

\subsubsection{Outlet boundary condition}

In the present model, outlet boundary condition is set to represent the convection dominated heat transfer across the outlet of the gas domain. The temperature gradient in the normal direction is zero. Thus, the boundary condition at the outlet in terms of heat flux can be written as:

$$
-\mathbf{n} \cdot \mathbf{q}=0
$$

The pressure boundary is set to specify the negligible normal stress to the flow at the outlet. We note that there is no tangential stress to the flow of fluid at the outlet of the gas domain, and the backflow is also suppressed.

$$
p=p_{\text {out }}
$$

Homogeneous Neumann conditions are also applied for the turbulence variables at the outlet,

$$
\left.\begin{array}{l}
\nabla k \cdot \mathbf{n}=0 \\
\nabla \varepsilon \cdot \mathbf{n}=0
\end{array}\right\}
$$

\subsubsection{Symmetry boundary condition}

Symmetry boundary condition is used to account similar physical processes on the two sides of boundary. It imposes zero heat flux across a boundary which means temperature on both sides of the boundary is equal. In a fluid-flow simulation, it prescribes no penetration and shear stresses. The symmetry boundary condition used in the present model can be written as-

$$
\left.\begin{array}{r}
-\mathbf{n} \cdot \mathbf{q}=0 \\
\mathbf{u} \cdot \mathbf{n}=0 \\
\nabla k \cdot \mathbf{n}=0 \\
\nabla \varepsilon \cdot \mathbf{n}=0
\end{array}\right\}
$$

\subsection{Performance of the heat exchanger}

We calculate following parameters including dimensionless terms to characterize the overall performance of the analyzed fin and tube heat exchanger design.

\subsubsection{Overall heat transfer coefficient}

Overall heat transfer coefficient $(U)$ is an important parameter to evaluate the performance of heat exchangers. It is defined as the total power exchanged between the geometric domains per unit heat transfer surface area per unit temperature difference [Cengel et al., 2012] and is expressed as:

$U=\frac{Q_{t}}{A_{t} \cdot \Delta T_{l m}}$

where, $Q_{t}[\mathrm{~W}]$ is the total power exchanged between the inner tube wall to the fluid flowing in gas domain. $A_{t}\left[\mathrm{~m}^{2}\right]$ is the total heat transfer surface area of the fin and tube. $\Delta T_{l m}[\mathrm{~K}]$ is log mean temperature difference.

\subsubsection{Colburn $j$-factor}

The gas-side heat transfer characteristic is often presented by Colburn $j$-factor $(j)$ [Taler and Ocłon, 2014; Li et al., 2015] as:

$j=\frac{N u}{\operatorname{Re} \cdot \operatorname{Pr}^{1 / 3}}$

where, $R e$ is Reynolds number and $P r$ is the Prandtl number.

\subsubsection{Flow resistance factor}

The mechanical performance can be evaluated in terms of flow resistance factor $(f)$ as a ratio of wall shear stress to kinetic energy of the flow.

$$
f=\frac{\Delta p}{\frac{1}{2} \rho_{g} \cdot u_{i n}^{2} \cdot\left(\frac{L_{g}}{D_{h}}\right)}
$$


where, $\Delta p[\mathrm{~Pa}]$ is the pressure difference across the gas domain, $\rho_{g}\left[\mathrm{~kg} / \mathrm{m}^{3}\right]$ and $u_{\text {in }}[\mathrm{m} / \mathrm{s}]$ is the density and inlet velocity of the gas, respectively. $L_{g}[\mathrm{~m}]$ is the length of the gas domain.

\subsubsection{Efficiency index}

The commonly used index for performance evaluation is the Efficiency index [Lu et al., 2013], which is a ratio of average Nusselt number to the pressure loss coefficient $\left(K_{g}\right)$.

Efficiency index $=\frac{N u}{K_{g}}=\frac{N u}{\left(\frac{\Delta p}{1 / 2 \rho_{g} \cdot u_{i n}^{2}}\right)}$

\section{Numerical procedure and solution}

Procedure of numerical simulation of a fin and tube heat exchanger design is described in this section. We used commercial multiphysics simulation software, COMSOL 5.1 based on FEM to solve the governing equations described in Section 3.2. With defined computational geometry (Figure 3), we start by setting initial values and model parameters (Table 1). Along with appropriate boundary conditions, governing equations for conjugate heat transfer are defined in all three domains (fin, tube and gas) and equations for turbulent flow are defined in gas domain. Heat transfer and fluid flow equations are then coupled together by the non-isothermal flow multiphysics. Mesh independence study is performed and a mesh of total 437519 elements is selected which is fine enough to resolve important physical phenomena accurately in a reasonable amount of time. To minimize the discontinuities and to capture sharp temperature gradients especially at the interface finer boundary layer mesh near to the interfaces is created. Figure 4 shows the refined FEM mesh used in the present study. The simulation was then ran for a computational time of nearly 30 minutes, which is sufficient to achieve steady state. The results obtained from the numerical model including the performance parameters are postprocessed for better understanding and visualization of fundamental phenomenological behavior. Table 2 lists the boundary condition values used to solve the model and achieve preliminary results.

Table 2. Boundary conditions values used to numerically solve the model.

\begin{tabular}{|l|c|c|c|}
\hline Description & Symbol & Value & Unit \\
\hline $\begin{array}{l}\text { Inner tube wall } \\
\text { temperature }\end{array}$ & $T_{w}$ & 453.15 & $\mathrm{~K}$ \\
\hline Inlet gas temperature & $T_{\text {in }}$ & 573.15 & $\mathrm{~K}$ \\
\hline Inlet gas velocity & $u_{\text {in }}$ & 9.60 & $\mathrm{~m} / \mathrm{s}$ \\
\hline Outlet gas pressure & $p_{\text {out }}$ & 0 & $\mathrm{~Pa}$ \\
\hline
\end{tabular}
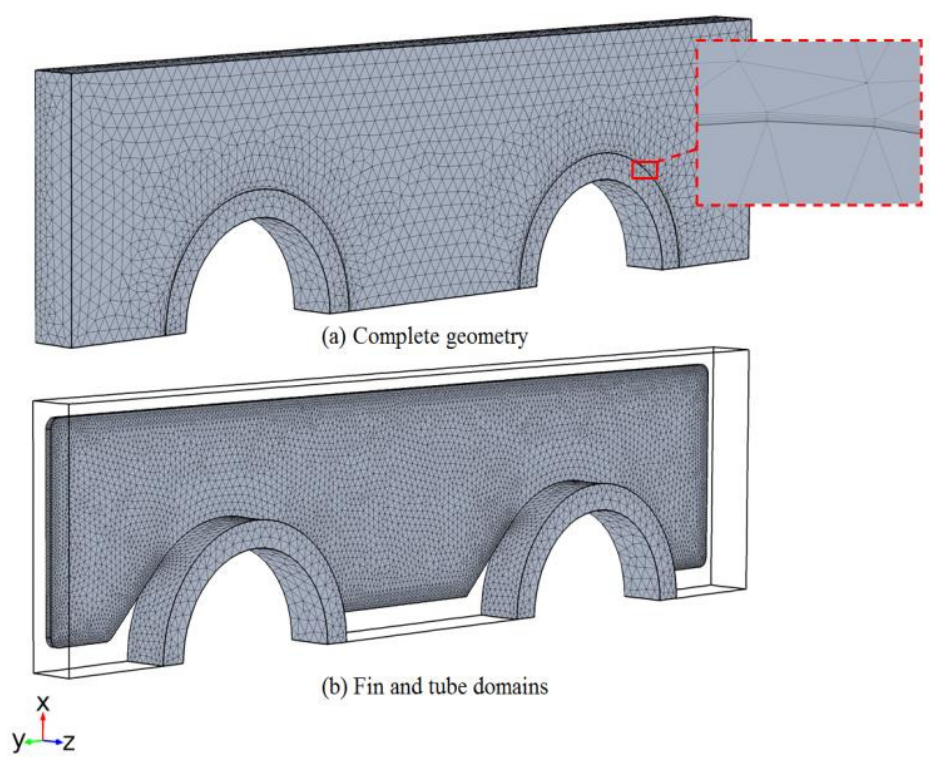

Figure 4. Mesh used in the numerical modeling.

\section{Results and discussion}

Since only half of the heat exchanger geometry is simulated in the present work, the results are represented for half of the geometry. Velocity profile influences the convective heat transfer to a great extent, hence it becomes important to analyze the velocity profile throughout the flow domain. Figure 5 represents the velocity profile of the flow in the gas domain. The inlet velocity profile can be clearly visualized where the gas enters the gas domain at a velocity of $9.6 \mathrm{~m} / \mathrm{s}$. It can be seen the gas flows with higher velocity near to the fin edges away from the tubes, while low velocity region generates between the tubes and at the bottom exit side of the tubes. Further velocity gradient across the length of gas domain can be clearly visualized using slice plot of the velocity profile as represented in Figure 6. The result is evident of frictional effect that develops along the length of the heat exchanger due to the tubes arrangement in an inline manner.

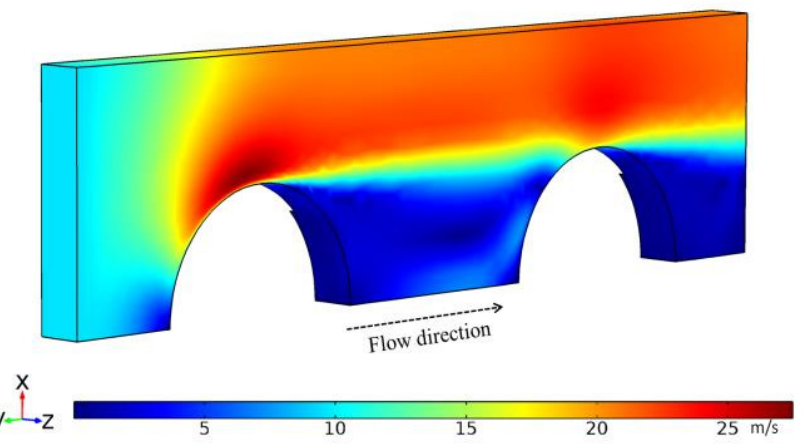

Figure 5. Velocity profile predicted in the gas domain 


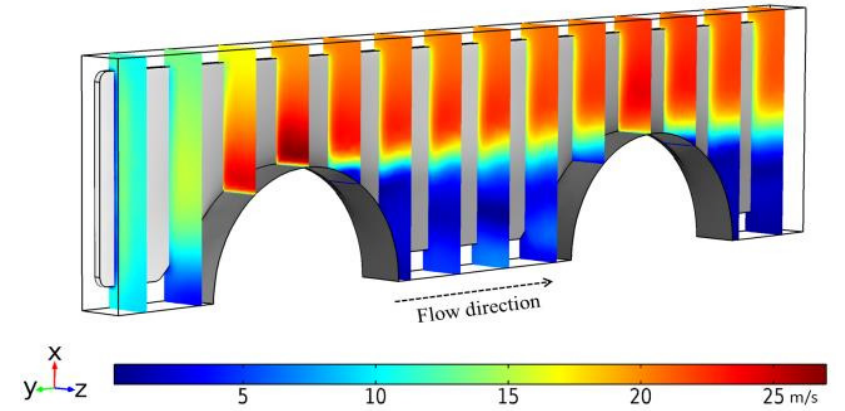

Figure 6. Slice plot for the velocity profile predicted in the gas domain

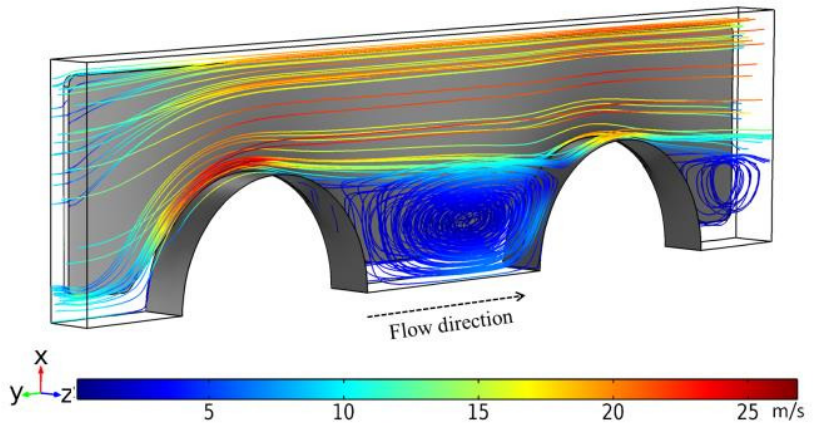

Figure 7. Streamline plot for the velocity profile predicted in the gas domain

The understanding of the flow passage within the domain is important to study the overall performance of the heat exchanger. Figure 7 depicts the velocity streamlines in the gas domain predicted by the model. Analysis of the velocity streamlines clearly shows the regions between the tubes and on the bottom exit side of the tube where the fluid recirculates to build a low or zero velocity zones. These zones prevent the effective heat transfer between the fluid and fin surface and thus degrades the overall performance of the heat exchanger.

Figure 8 shows the temperature distribution in the entire computational geometry predicted by the model. The gas enters into the gas domain at a temperature $573.15 \mathrm{~K}$ and is cooled down as it flows from the inlet to the outlet. The figure demonstrates the simultaneous process of conjugate heat transfer and turbulent flow. The heat is transferred from the flowing gas to fin and tube surface by convection and from outer surface fin and tube to the inner wall of the tube by conduction. The temperature gradients in Figure 8 clearly illustrate the strong influence of velocity profile on heat transfer in the heat exchanger design.

The temperature distribution in the gas domain is predicted by the model and is shown as slice plot in Figure 9. As observed, the gas has higher temperature at the inlet and on the fin edge side while lower temperature region exists between the tubes. The reason attributes to the developed low velocity region, which affects the heat transfer in the heat exchanger.

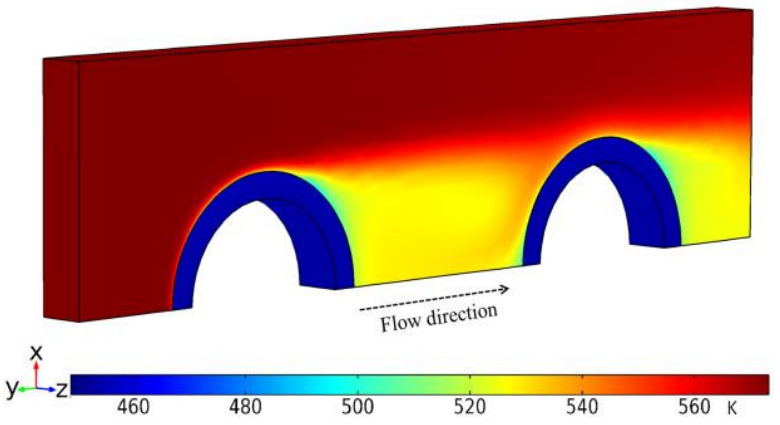

Figure 8. Temperature distribution predicted in the computational domain

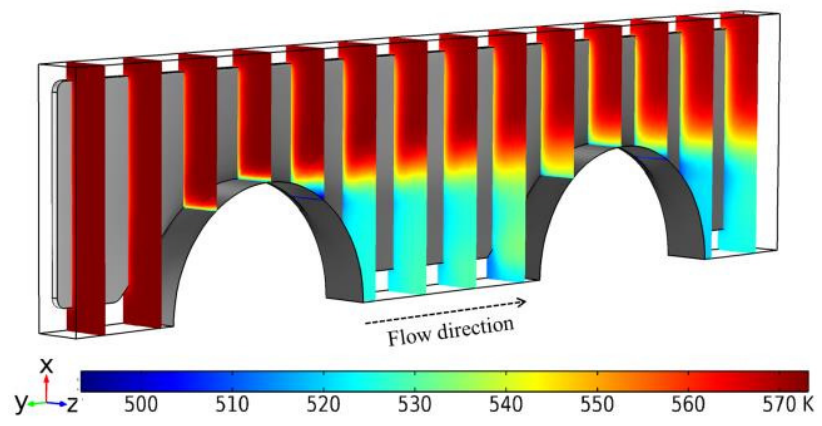

Figure 9. Slice plot for the temperature distribution predicted in the gas domain

Figure 10 depicts the predicted temperature distribution in the fin and tube domains. The high temperature can be seen at the inlet region from where hot gas enters into the gas domain. The temperature decreases along the length of the fin due to conjugate heat transfer. It is observed that the temperature gradients are high in the regions near to the fin base, where fin comes in contact with the tubes. These regions can be visualized as the regions of effective heat transfer. However, middle region of the fin length up to outer edge shows low temperature gradients and thus is not effective to contribute significantly in the overall heat transfer process. The reason attributes to the high velocity in this region that (Figure 6 and 7) does not allow the hot gas to stay for sufficient time for allowing better heat transfer.

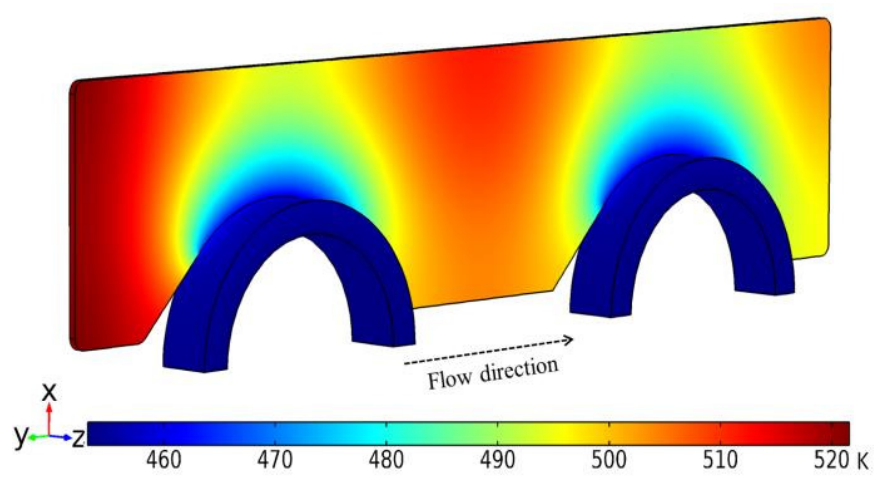

Figure 10. Temperature distribution predicted in the fin and tube domains 


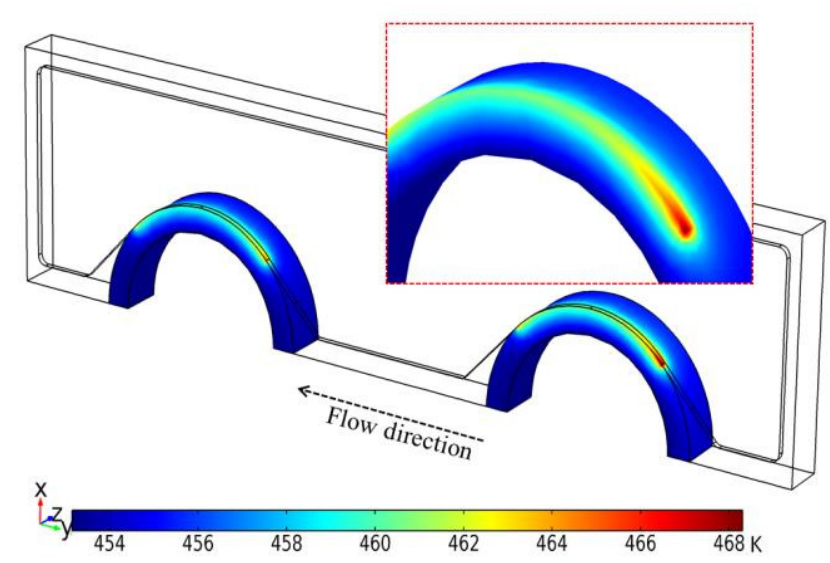

Figure 11. Contour plane plot of the conductive heat flux in fin and tube domain

The results are evident from the Figure 11, which shows temperature distribution in tube domain. It is clearly seen that the temperature gradients are high near to the contact interface of the fin and tubes. Higher temperature difference at the fin base leads to higher conductive heat flux transferred from the fin to the tube in this region. Temperature distribution in the first tube in the gas flow direction can be observed from the insert in Figure 11.

To help understand the results, vector plot for gas velocity field with temperature variations on fin and outer tube wall can be seen together in Figure 12. The direction of the arrows represents gas flow from the inlet to the outlet. The figure shows that how velocity vector moves around to create inefficient low heat transfer zone between the tubes and bottom exit side of the tube. In addition, temperature variations in the fin, tube and gas domain can also be seen simultaneously in the Figure 12.

The performance of the heat exchanger design is evaluated in terms of performance parameters defined in Section 3.4. Table 3 summarizes the values of performance parameters (Equations (17)-(20)) computed using the numerical model.

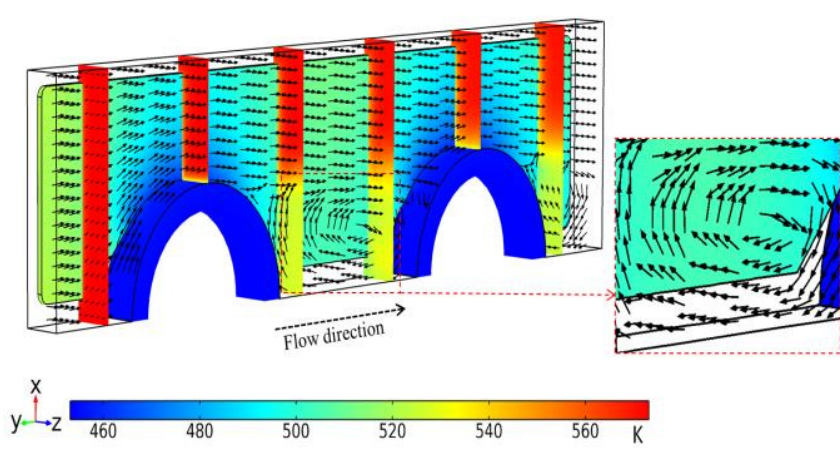

Figure 12. Vector plot for the velocity profile in gas domain with temperature distribution in fin and gas domain
Table 3. Performance parameters calculated from the numerical model

\begin{tabular}{|l|l|l|}
\hline Performance parameter & Value & Unit \\
\hline $\begin{array}{l}\text { Overall heat transfer } \\
\text { coefficient }\end{array}$ & 41.499 & $\mathrm{~W} / \mathrm{m}^{2} \mathrm{~K}$ \\
\hline Colburn $j$-factor & 0.0051 & Dimensionless \\
\hline Flow resistance factor & 0.8146 & Dimensionless \\
\hline Efficiency index & 3.474 & Dimensionless \\
\hline
\end{tabular}

\section{Conclusion}

Multiphysics 3D numerical modeling of a fin and tube heat exchanger is performed. The model incorporates coupled heat transfer and turbulent flow to analyze the physical phenomenon in the heat exchanger operation. The model has a capability to predict the steady state temperature and velocity profiles, and thus predicts the overall performance of the heat exchanger design. The overall heat transfer coefficient for the heat exchanger design is calculated to be $41.499 \mathrm{~W} / \mathrm{m}^{2} \mathrm{~K}$ with an efficiency index of 3.474. The model predictions help to identify the areas where heat transfer is intensive and those where it is ineffective for waste heat recovery including bottlenecks to heat transfer within the heat exchanger which can be overcome by optimization of the design. Studies to optimize the weight of the heat exchanger will be conducted and a separate research article will be communicated in future. Moreover, developed model delivers vital guiding information to support its feasibility for large scale application in realistic conditions.

\section{Acknowledgement}

The work presented in this paper is a part of research project THERMCYC-Advanced thermodynamic cycles utilising low-temperature heat sources (No. 13050036B).

\section{References}

A. Erek, B. O"zerdem, L. Bilir and Z. Ilken. Effect of geometrical parameters on heat transfer and pressure drop characteristics of plate fin and tube heat exchangers. Applied Thermal Engineering, 25: 2421-2431, 2005.

A. Jacobi and R. Shah. Heat transfer surface enhancement through the use of longitudinal vortices: a review of recent progress. Experimental Thermal Fluid Science, 11: 295309, 1995

B. Gong, L. Wang and Z. Lin. Heat transfer characteristics of a circular tube bank fin heat exchanger with fins punched curve rectangular vortex generators in the wake regions of the tubes. Applied Thermal Engineering, 75: 224-238, 2015.

B. Kundu and K. Lee. Thermal design of an orthotropic flat fin in fin-and-tube heat exchangers operating in dry and wet environments. International Journal of Heat and Mass Transfer, 54:5207-5215, 2011.

C. C. Wang, W. H. Tao and C. J. Chang. An investigation of the airside performance of the slit fin-and-tube heat 
exchangers. International Journal of Refrigeration, 22: 595-603, 1999.

C. Habchi, S. Russeil, D. Bougeard, J. Harion, T. Lemenand, D. D. Valle and H. Peerhossaini. Enhancing heat transfer in vortex generator-type multifunctional heat exchangers. Applied Thermal Engineering, 38:14-25, 2012.

D. C. Wilcox. Turbulence Modeling for CFD. 2nd ed., DCW Industries, 1998.

D. Kuzmin, O. Mierka and S. Turek. On the Implementation of the k- $\varepsilon$ Turbulence Model in Incompressible Flow Solvers Based on a Finite Element Discretization. International Journal of Computing Science and Mathematics, 1(2-4): 193-206, 2007.

D. Taler and P. Ocłon. Thermal contact resistance in plate fin-and-tube heat exchangers determined by experimental data and CFD simulations. International Journal of Thermal Science, 84: 309-322, 2014.

J. Cobian- Iñiguez, A. Wu, F. Dugast and A. Pacheco-Vega. Numerically-based parametric analysis of plain fin and tube compact heat exchangers. Applied Thermal Engineering, 86: 1-13, 2015.

J. Y. Jang and J. Y. Yang. Experimental and numerical analysis of the thermal hydraulic characteristics of elliptic finned-tube heat exchangers. Heat Transfer Engineering, 19(4): 55-67, 1998.

L. A. O. Rocha, F. E. M. Saboya and J. V. C. Vargas. A comparative study of elliptical and circular sections in oneand two-row tubes and plate fin heat exchangers, International Journal of Heat and Fluid Flow, 18(2): 247252, 1997.

L. H. Tang, Z. Min, G. N. Xie and Q. W. Wang. Fin Pattern Effects on Air-Side Heat Transfer and Friction Characteristics of Fin-and-Tube Heat Exchangers with Large Number of Large-Diameter Tube Rows. Heat Transfer Engineering, 30(3): 171-180, 2009.

L. Li, X. Du, Y. Zhang, L. Yang and Y. Yang. Numerical simulation on flow and heat transfer of fin-and-tube heat exchanger with longitudinal vortex generators. International Journal of Thermal Sciences, 92: 85-96, 2015.

L. M. Chang, L. B. Wang, K. W. Song and D. L. Sun. Numerical study of the relationship between heat transfer enhancement and absolute vorticity flux along main flow direction in a channel formed by a flat tube bank fin with vortex generators. International Journal of Heat and Mass Transfer, 52: 1794-1801, 2009.

M. Fiebig. Embedded vortices in internal flow: heat transfer and pressure loss enhancement. International Journal of Heat and Fluid Flow, 16(5):376-388, 1995.

M. Hatami, D. D.Ganji and M. Gorji-Bandpy. Numerical study of finned type heat exchangers for ICEs exhaust waste heat recovery. Case Studies in Thermal Engineering 4:53-64, 2014a.

M. Hatami, D. D. Ganji and M. Gorji-Bandpy. A review of different heat exchangers designs for increasing the diesel exhaust waste heat recovery. Renewable and Sustainable Energy Reviews, 37:168-181, 2014b.

M. Hatami, D. D. Ganji and M. Gorji-Bandpy. CFD simulation and optimization of ICEs exhaust heat recovery using different coolants and fin dimensions in heat exchanger. Neural Computing and Applications, 25: 20792090, 2014c.

M. S. Mon and U. Gross. Numerical study of fin-spacing effects in annular-finned tube heat exchangers. International Journal of Heat and Mass Transfer, 47: 1953-1964, 2004.

P. Chu, Y. L. He, Y. G. Lei, L. T. Tian and R. Li. Threedimensional numerical study on fin-and-oval-tube heat exchanger with longitudinal vortex generators. Applied Thermal Engineering, 29: 859-876, 2009.

R. Borrajo-Peláez, J. Ortega-Casanova and J. M.CejudoLópez. A three-dimensional numerical study and comparison between the air side model and the air/water side model of a plain fin-and-tube heat exchanger. Applied Thermal Engineering, 30: 1608-1615, 2010.

R. S. Matos, J. V. C. Vargas, T. A. Laursen and T. A. Bejan. Optimally staggered finned circular and elliptic tubes in forced convection. International Journal of Heat and Mass Transfer, 47(13): 47-59, 2004.

S. Lopata and P. Ocłon. Numerical study of the effect of fouling on local heat transfer conditions in a hightemperature fin-and-tube heat exchanger. Energy doi:10.1016/j.energy.2015.03.048, 2015

T. A. Ibrahim and A. Gomma. Thermal performance criteria of elliptic tube bundle in crossflow. International Journal of Thermal Science, 48(11): 2148-2158, 2009.

T. J. Lu, F. Xu and T. Wen. Thermo-fluid behaviour of periodic cellular metals. Springer Heidelberg New York Dordrecht London (ISBN 978-3-642-33523-5), 2013.

V. P. Malapure, S. K. Mitra and A. Bhattacharya. Numerical investigation of fluid flow and heat transfer over louvered fins in compact heat exchanger. International Journal of Thermal Sciences, 46:199-211, 2007.

W. Yaïci, M. Ghorab and E. Entchev. 3D CFD analysis of the effect of inlet air flow maldistribution on the fluid flow and heat transfer performances of plate-fin-and-tube laminar heat exchangers. International Journal of Heat and Mass Transfer, 74:490-500, 2014.

Y. A. Cengel, J. M. Cimbala and R. H. Turner. Fundamentals of thermal-fluid sciences, fourth edition in SI units. McGraw-Hill, 2012.

Y. B. Tao, Y. L. He, J. Huang, Z. G. Wu and W.Q. Tao. Numerical study of local heat transfer coefficient and fin efficiency of wavy fin-and-tube heat exchangers. International Journal of Thermal Sciences, 46: 768-778, 2007.

Y. Chen, M. Fiebig and N. K. Mitra. Conjugate heat transfer of a finned oval tube part a: flow patterns, Numerical Heat Transfer, Part A: Applications. An International Journal of Computation and Methodology, 33(4): 371-385, 2007. 